\title{
Development and validation of a prediction rule for early discharge of low-risk emergency department patients with potential ischemic chest pain
}

\author{
Frank Xavier Scheuermeyer, MD, MHSc*; Hubert Wong, PhD*; Eugenia Yu, MSc*; \\ Barb Boychuk, RN*; Grant Innes, MD*; Eric Grafstein, $\mathrm{MD}^{\dagger}$; Kenneth Gin, $\mathrm{MD}^{\ddagger}$; Jim Christenson, MD*
}

\section{ABSTRACT}

Objectives: Current guidelines emphasize that emergency department (ED) patients at low risk for potential ischemic chest pain cannot be discharged without extensive investigations or hospitalization to minimize the risk of missing acute coronary syndrome (ACS). We sought to derive and validate a prediction rule that permitted 20 to $30 \%$ of ED patients without ACS safely to be discharged within 2 hours without further provocative cardiac testing.

Methods: This prospective cohort study enrolled 1,669 chest pain patients in two blocks in 2000-2003 (development cohort) and 2006 (validation cohort). The primary outcome was 30 -day ACS diagnosis. A recursive partitioning model incorporated reliable and predictive cardiac risk factors, pain characteristics, electrocardiographic findings, and cardiac biomarker results.

Results: In the derivation cohort, 165 of 763 patients (21.6\%) had a 30-day ACS diagnosis. The derived prediction rule was $100.0 \%$ sensitive and $18.6 \%$ specific. In the validation cohort, 119 of 906 patients $(13.1 \%)$ had ACS, and the prediction rule was $99.2 \%$ sensitive $(95 \% \mathrm{Cl} 95.4-100.0)$ and $23.4 \%$ specific (95\% Cl 20.6-26.5). Patients have a very low ACS risk if arrival and 2-hour troponin levels are normal, the initial electrocardiogram is nonischemic, there is no history of ACS or nitrate use, age is $<50$ years, and defined pain characteristics are met. The validation of the rule was limited by the lack of consistency in data capture, incomplete follow-up, and lack of evaluation of the accuracy, comfort, and clinical sensibility of this clinical decision rule.

Conclusion: The Vancouver Chest Pain Rule may identify a cohort of ED chest pain patients who can be safely discharged within 2 hours without provocative cardiac testing. Further validation across other centres with consistent application and comprehensive and uniform follow-up of all eligible and enrolled patients, in addition to measuring and reporting the accuracy of and comfort level with applying the rule and the clinical sensibility, should be completed prior to adoption and implementation.

\section{RÉSUMÉ}

Objectif: D'après les lignes directrices actuelles, on ne peut renvoyer du service des urgences (SU) les patients qui souffrent de douleurs thoraciques de nature probablement ischémique mais qui connaissent un faible risque de maladie cardiaque sans d'abord leur faire subir une exploration approfondie ou sans les hospitaliser afin de réduire le plus possible le risque de syndrome coronarien aigu (SCA) passé inaperçu. Aussi avons-nous tenté d'élaborer et de valider une règle prévisionnelle qui permettrait de renvoyer en toute sécurité de 20 à $30 \%$ des patients ne souffrant pas d'un SCA au cours des 2 heures suivant leur arrivée au SU, et ce, sans leur faire subir d'autres épreuves d'exploration cardiaque.

Méthode: II s'agit d'une étude prospective de cohortes à laquelle ont participé 1,669 patients souffrant de douleurs thoraciques; deux groupes ont été formés: I'un, de 2000 à 2003 (cohorte d'élaboration); I'autre, en 2006 (cohorte de validation). Le principal critère d'évaluation était la pose d'un diagnostic de SCA au bout de 30 jours. Étaient incorporés dans un modèle de partitionnement récursif des facteurs de risque fiables et prévisionnels de maladie cardiaque, les caractéristiques de la douleur, les signes électrocardiographiques, et les valeurs des biomarqueurs cardiaques.

Résultats: Dans la cohorte d'élaboration, un diagnostic de SCA avait été posé au bout de 30 jours chez 165 patients sur 763 (21.6\%); la règle prévisionnelle qui en avait découlé avait une sensibilité de $100.0 \%$ et une spécificité de $18.6 \%$. Dans la cohorte de validation, un diagnostic de SCA avait été posé chez 119 patients sur 906 (13.1\%); la règle prévisionnelle avait une sensibilité de 99.2\% (IC à 95\%: 95.4-100.0) et une spécificité de

From the *Department of Emergency Medicine, St. Paul's Hospital and the University of British Columbia, Vancouver, BC; †Division of Emergency Medicine, Foothills Hospital and the University of Calgary, Calgary, AB; and ¥Division of Cardiology, Department of Medicine, Vancouver Hospital and the University of British Columbia, Vancouver, BC.

Correspondence to: Dr. Frank Xavier Scheuermeyer, Department of Emergency Medicine, St. Paul's Hospital, 1081 Burrard Street, Vancouver, BC V6Z 1Y6; frankscheuermeyer@yahoo.ca.

This article has been peer reviewed 
23.4\% (IC à 95\%: 20.6-26.5). Ainsi, les patients connaissent un très faible risque de SCA si les taux de troponine à l'arrivée et au bout de 2 heures sont normaux; si l'électrocardiogramme ne montre pas de signe d'ischémie; s'il n'y a pas d'antécédents de SCA ou d'emploi de dérivés nitrés; si l'âge est inférieur à 50 ans; et si les caractéristiques de la douleur concordent avec celles qui ont été établies. Cependant, le manque d'uniformité dans la collecte des données, les suivis incomplets, et une évaluation insuffisante de la précision, de la facilité d'utilisation, et de la sensibilité clinique de cette règle décisionnelle en ont amoindri la validation.

Conclusions: La "règle de Vancouver» sur les douleurs thoraciques permet de cerner une cohorte de patients

Over 8 million patients present to US emergency departments (EDs) annually with potential ischemic chest pain, ${ }^{1}$ and these may account for $25 \%$ of hospital admissions. $^{2}$ In Canada, estimates from Ontario (Dr. Michael Schull, Sunnybrook Hospital and the University of Toronto, personal communication, August 20, 2012) and Vancouver (Dr. Eric Grafstein, St. Paul's Hospital and the University of British Columbia, personal communication, August 20,2012) suggest over 600,000 annual ED visits. Between 2 and $5 \%$ of patients with acute coronary syndrome (ACS) may be discharged with an incorrect non-ACS diagnosis and no further follow-up, ${ }^{3,4}$ leading to adverse patient outcomes and medicolegal costs..$^{5}$ As initial electrocardiogram (ECG) and biomarker results may lack sensitivity, further investigation is usually required. Although most patients are at low risk, many centres attempt to maximize diagnostic sensitivity by applying comprehensive ruleout protocols involving prolonged monitoring, serial cardiac investigations, provocative or invasive testing, and often admission to observation or coronary care units $^{6-8}$; a recent study from Minnesota reported that nearly all chest pain patients were admitted to hospital. ${ }^{9}$

Prediction rules have been developed for ACS risk stratification, ${ }^{9-16}$ but few $^{14-16}$ have been validated and none widely accepted. The 2007 American College of Cardiology/American Heart Association (ACC/AHA) guidelines, ${ }^{6}$ the 2005 AHA guidelines, ${ }^{7}$ and a recent AHA statement ${ }^{8}$ do not discuss early discharge of lowrisk patients without additional testing. Rather, for any potential ACS patient, they recommend 6 to 12 hours of observation, serial ECG and biomarker testing, and additional confirmatory testing. This approach drives high resource use and increases ED crowding, ${ }^{17}$ potentially leading to substandard care and increased recurrent myocardial infarctions. ${ }^{18,19}$ souffrant de douleurs thoraciques mais susceptibles d'être renvoyés en toute sécurité au cours des 2 heures suivant leur arrivée au SU, sans leur faire subir des épreuves d'exploration cardiaque. Toutefois, il faudrait procéder à une validation de la règle, avant son adoption et sa mise en œuvre, dans d'autres centres hospitaliers, assortie d'une application uniforme de la ligne de conduite et d'un suivi général et homogène de tous les patients admissibles et inscrits; à cela s'ajouteraient une mesure de la précision de la règle et du degré de facilité de son application ainsi qu'une évaluation de sa sensibilité clinique.

Keywords: cardiology, outcomes, patient safety
A sensitive ACS prediction rule would help physicians identify low-risk patients who can be managed without extensive investigations. An acceptable rule could decrease lengths of stay and ED crowding, decrease patient anxiety, limit expensive or invasive testing, and preserve hospital resources for higher-risk patients.

Our objective was to (1) develop ${ }^{20}$ a clinical decision rule based on data available within 2 hours of arrival that would identify low-risk ED chest pain patients suitable for early discharge without further provocative cardiac testing and (2) validate this rule with a separate cohort of patients. Based on a previous Canadian survey of emergency physicians, ${ }^{21}$ the goal was for ACS sensitivity to exceed $98 \%$ while identifying the maximum number of low-risk patients who did not require hospitalization or prolonged testing.

\section{MATERIALS AND METHODS}

\section{Study design and setting}

St. Paul's Hospital is a university-affiliated urban tertiary care centre with 60,000 annual ED visits during the study period. The derivation cohort was enrolled in two separate periods between June 29, 2000, and January 24, 2003. The prospective validation cohort enrolled patients between February 1, 2006, and September 29, 2006. Accepted methodology for clinical prediction rule development was followed. ${ }^{22,23}$

\section{Patients}

Consenting patients presenting to the ED with a chief complaint of anterior or lateral chest pain of a potential ischemic nature were eligible for enrolment. Patients typically received a Canadian Emergency Department 
Information Systems ${ }^{24}$ (a standardized, validated form of triage complaints) designation of "chest pain, cardiac features" when they presented at the triage desk and were typically assigned a Canadian Triage and Acuity Scale $^{25}$ (CTAS, a reliable and validated 5-point triage system) level 2 (urgent physician assessment) code. Patients were excluded if they were less than 25 years old; had a clear traumatic or radiologically evident alternate cause of chest pain; were previously enrolled within 30 days; or had a terminal illness, debilitating communication problem, or no telephone contact. The University of British Columbia and Providence Health Care Research Ethics Board approved this study.

\section{Sample size}

The sample size was calculated based on the following premises: (1) an ACS rate of $\approx 23 \%$ estimated for both the development and validation cohorts based on previous site data; (2) setting the lower goal of the $95 \%$ confidence interval for sensitivity at $95 \%$; and (3) assuming that the true sensitivity of the rule is 0.98 , around 187 ACS cases requiring an overall enrolment of 815 patients were required to exceed this lower bound of $95 \%$ probability. ${ }^{20}$

\section{Development cohort}

Standard reporting guidelines for risk stratification of ED chest pain patients were followed. ${ }^{26}$ Procedures used to enrol the development cohort and derive the prediction rule are described in detail in a previous publication..$^{20}$ Briefly, research assistants who were blinded to patient outcomes screened and enrolled consecutive patients from 7 am to $11 \mathrm{pm}$. As patients also underwent nonstandard investigations such as serum myoglobin, they consented to both new investigations and 30-day telephone follow-up. During the initial 2 hours of the ED stay, assistants recorded vital signs and ensured that serial ECGs and troponin $T$ assays (Roche Elecsys, Hoffman LaRoche, Laval, QC; 99th percentile reference limit $>0.01 \mathrm{ng} / \mathrm{mL}$, lower limit of detection $0.01 \mathrm{ng} / \mathrm{mL}, 10 \%$ coefficient of variation $0.03 \mathrm{ng} / \mathrm{mL}$ ) were performed. Ninety-seven predictor variables were screened and recorded for each patient (Appendix 1). Interrater reliability was investigated by having a second emergency physician, blinded to the assessment of the first physician and to outcomes, independently assess a $10 \%$ convenient sample to generate kappa values.

\section{ECG assessment}

A single trained emergency physician blinded to both predictor variables and outcomes independently reviewed each ECG for rate, rhythm, and ischemic changes. These were defined as ST segment elevation $>$ $2 \mathrm{~mm}$ in two consecutive precordial leads or $>1 \mathrm{~mm}$ in two consecutive inferior or lateral leads, or the following prespecified potential ischemic changes: any ST depression $>2 \mathrm{~mm}$ in $\mathrm{V} 1$ or $\mathrm{V} 2$ (denoting posterior infarction), ST depression $>0.5 \mathrm{~mm}$ in two contiguous leads, Q waves in two contiguous leads or T-wave inversion in two contiguous leads, left ventricular hypertrophy, paced rhythm, and left bundle branch block (LBBB). ${ }^{27}$ Interrater reliability was assured by having a second emergency physician reviewer, who was blinded to the assessment of the first physician, assess the first $20 \%$ of ECGs for an overall dichotomous determination of "ischemic features" or "no ischemic changes."

\section{Validation cohort}

Assistants enrolled consecutive patients from 7 am to $11 \mathrm{pm}$. To maximize nighttime enrolment, patients who arrived after $11 \mathrm{pm}$ but were discharged before 7 am were consented and enrolled postdischarge via telephone contact. Data collection was reduced to 53 potential predictor variables for each validation patient based on reliability and correlation measures from the derivation set (see Appendix 1).

\section{Re-exploration of development cohort}

A previously derived rule from the same cohort described in this article was $99.4 \%$ sensitive and $32.5 \%$ specific for ACS detection. ${ }^{20}$ However, this rule used the cardiac biomarker creatinine kinase-muscle/ brain isomer (CK-MB) as a key decision point. Early in the rule validation phase, CK-MB fell out of favour, and our institution discontinued its availability, making validation impossible. A new rule was derived based on the same development cohort, incorporating the 53 predictor variables discussed above and troponin as the key biomarker instead of CK-MB.

\section{0-day follow-up (both cohorts)}

Research staff contacted patients via telephone to review medications and document physician or hospital visits 
that occurred within 30 days of the index ED visit. Test results (additional ECGs and troponin testing, provocative testing [exercise stress testing, radionuclide imaging, angiography]) were also recorded. If patient contact was unsuccessful, all records from hospital visits within the region were obtained to establish or refute a 30-day diagnosis of ACS. The following predefined adverse events (AEs) occurring within 30 days were identified: tachycardia or bradycardia requiring medical intervention; respiratory failure requiring assisted ventilations; pulmonary embolism; aortic dissection or aneurysm; new congestive heart failure requiring intravenous medications; hypotension requiring vasoactive agents or an intra-aortic balloon pump; chest compressions; percutaneous coronary intervention; or coronary artery bypass grafting. The enrolled patients were cross-checked with the provincial death registry to identify individuals who had died within 30 days.

\section{Outcome measures}

Emergency physician assessors who were blinded to all predictor variables used predetermined criteria (Table 1) to establish one of the following mutually exclusive 30-day diagnoses: acute myocardial infarction (AMI), unstable angina (UA), or no ACS. ${ }^{6,27}$ A 30-day diagnosis of ACS (AMI or UA) was the primary outcome.

All potentially misclassified outcomes were independently reviewed by two cardiologists blinded to the initial classification, and discordant adjudications were resolved by the principal investigator (J.C.). Potentially misclassified outcomes were defined as (1) every death or ACS diagnosis without ECG changes or cardiac

\footnotetext{
Table 1. Outcome definitions

Acute myocardial infarction

Troponin $T>$ 99th percentile of the upper reference limit with a change of $20 \%$ between serial measurements

ECG changes consistent with acute MI, including

New pathologic $Q$ waves

New LBBB

New ST elevation at $\mathrm{J}$ point in two contiguous leads: $2 \mathrm{~mm}$ in precordial leads or $1 \mathrm{~mm}$ in other leads (in the absence of LBBB or left ventricular hypertrophy)

Death with no other cause evident

Unstable angina

Coronary angiogram with $>70 \%$ lesion

Revascularization with either percutaneous coronary intervention or coronary artery bypass grafting

ECG = electrocardiogram; LBBB = left bundle branch block; $\mathrm{MI}=$ myocardial infarction.
}

biomarker elevation, (2) patients not classified as ACS, but having a troponin elevation or ECGs indicative of ACS.

\section{Data analysis}

Data were collected on specially designed study forms and entered into an Oracle database (Oracle Corporation, Redwood City, CA). All potential predictors and 30-day outcomes were entered twice into the database for each case, and discrepancies were reviewed to ensure transcription accuracy in the final database. Descriptive summaries were produced using SAS (SAS Institute Inc., Cary, NC). The prediction rule was derived with the S-Plus (Insightful Corp, Seattle, WA) "rpart" recursive partitioning program. To maximize sensitivity, a 10:1 loss was specified for missed ACS patients compared to missed non-ACS patients. The eligibility criteria for a variable to be considered for inclusion in the rule were a univariate association with the outcome of $p<0.20$ and adequate interrater reliability for subjective variables (e.g., elements from the history and physical examination) prespecified as a lower confidence boundary for the kappa coefficient of $>0.4$. Eligible variables were entered into the recursive partitioning program, and multiple rules were derived. Investigators scrutinized each rule to determine clinical relevance, and the simplest rule with a sensitivity of at least $98 \%$ was chosen. Sensitivity, specificity, and predictive values were obtained for rule development but not confidence intervals (see above). Results obtained from the validation cohort were then placed into the rule, and sensitivity, specificity, and predictive values along with their confidence intervals were calculated.

\section{RESULTS}

Figure 1 graphically displays the 763 patients analyzed in the development cohort and 906 in the validation cohort. Although 73 patients were lost to follow-up, noncontact alternative sources verified that none had a 30-day regional ED visit or appeared on the provincial death registry. Two emergency physicians, blinded to predictors and outcomes, independently assessed 150 ECGs (19.7\%) from the derivation cohort to obtain interrater reliability for "ischemic changes"; a kappa value of 0.83 was obtained.

Table 2 displays patient characteristics and outcomes for the derivation $(n=763)$ and validation $(n=906)$ 
Development cohort

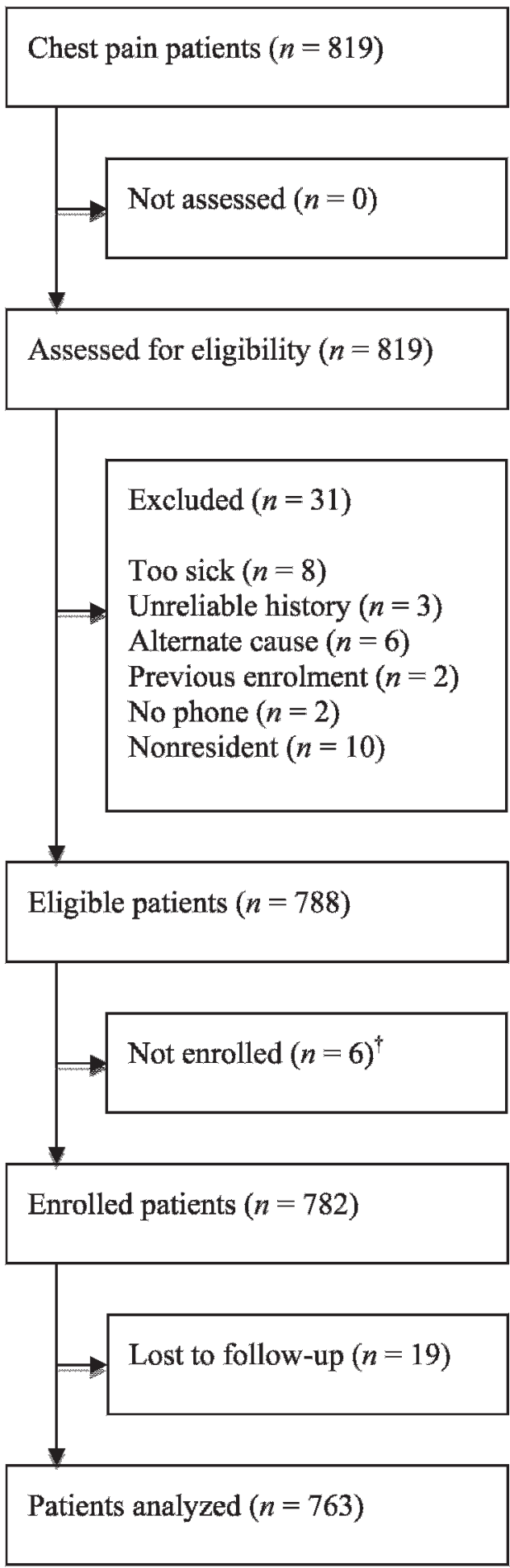

Validation cohort

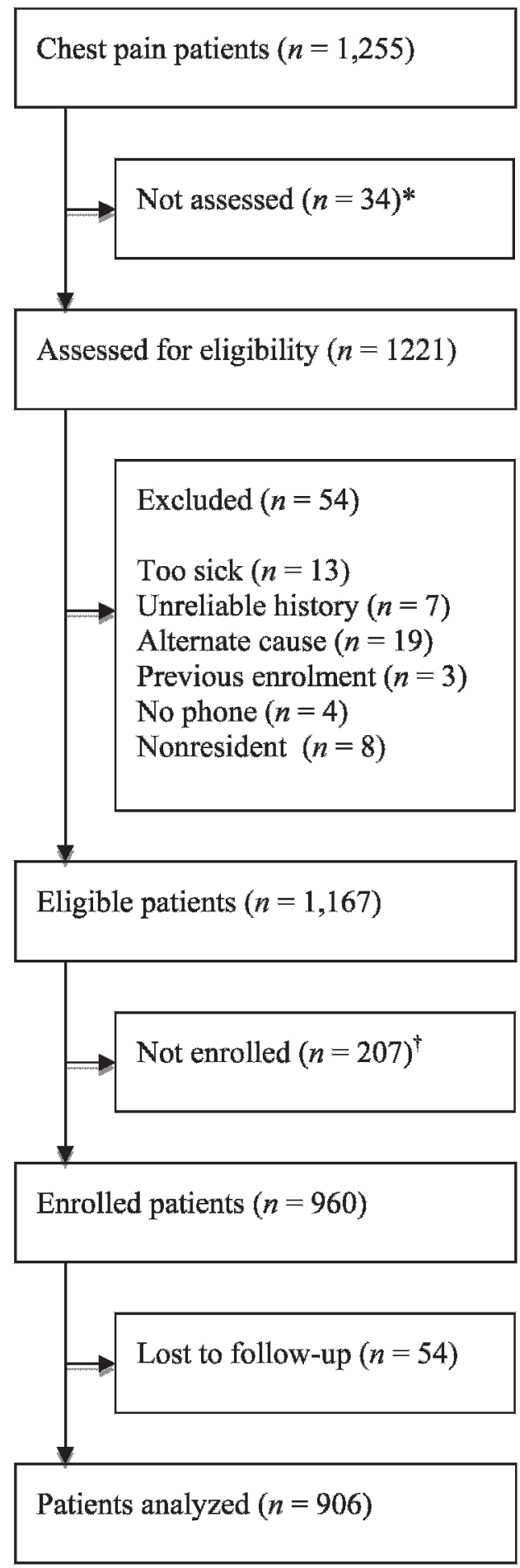

Figure 1. Study flow diagram. *Not assessed due to nighttime arrival. See Methods in the text. ${ }^{\dagger}$ Not enrolled in rule since troponin was not measured. See Limitations in the text. 


\begin{tabular}{|c|c|c|}
\hline Variable & Derivation cohort ( $n=763$ ) & Validation cohort ( $n=906$ ) \\
\hline Mean age (SD) & $58(14)$ & $60(15)$ \\
\hline Male sex, $n(\%)$ & $473(62.0)$ & $555(61.2)$ \\
\hline Arrived by EMS, $n(\%)$ & $238(31.2)$ & $280(30.9)$ \\
\hline \multicolumn{3}{|l|}{ Initial vital signs, mean (SD) } \\
\hline Heart rate (beats/min) & $81.2(20.0)$ & $80.2(19.5)$ \\
\hline Systolic BP (mm Hg) & $144.1(27)$ & $143.2(26.5)$ \\
\hline Respiratory rate (breaths/min) & $18.8(3.4)$ & $18.6(3.2)$ \\
\hline \multicolumn{3}{|l|}{ Risk factors, $n(\%)$} \\
\hline Diabetes & $128(16.8)$ & $147(16.2)$ \\
\hline Hypertension & $293(38.4)$ & $386(42.6)$ \\
\hline Hyperlipidemia & $278(36.4)$ & $286(31.5)$ \\
\hline \multicolumn{3}{|l|}{ Cardiac, $n(\%)$} \\
\hline Previous ACS & $311(40.8)$ & $267(29.4)$ \\
\hline Abnormal initial ECG & $229(30.0)$ & $268(29.6)$ \\
\hline \multicolumn{3}{|l|}{ 30-day outcomes, $n(\%)$} \\
\hline AMI & $77(10.1)$ & $39(4.3)$ \\
\hline Definite unstable angina & $88(11.6)$ & $80(8.8)$ \\
\hline Possible unstable angina & $29(3.8)$ & $21(2.3)$ \\
\hline No ACS but $A E$ & $32(4.1)$ & $23(2.5)$ \\
\hline No ACS, no AE & $537(69.8)$ & $766(84.5)$ \\
\hline Death & $9(1.1)$ & $5(0.6)$ \\
\hline
\end{tabular}

cohorts. The derivation cohort had 77 patients with AMI (10.1\%) and 88 patients with definite UA $(11.5 \%)$, whereas the validation cohort had 39 patients with AMI (4.3\%) and 80 patients $(8.8 \%)$ with definite UA. There were 11 outcomes requiring adjudication, 6 in the derivation cohort and 5 in the validation cohort. The sample size was not achieved for either cohort due to a reduction in the ACS incidence rate (21.6\% development cohort and $13.1 \%$ validation cohort), resulting in insufficient funding to enrol sufficient patients.

Variables used in the recursive partitioning algorithm are illustrated in Appendix 1; those selected for entry into the recursive partitioning algorithm are displayed with respective univariate statistics in Appendix 2. Interrater reliability measures (kappa) for clinically subjective variables were as follows: pain radiating to the neck, 0.68 (95\% CI 0.54-0.83); pain radiating to the jaw, 0.81 (95\% CI 0.69-0.93); pain radiating to the left arm, 0.79 (95\% CI $0.67-$ 0.91 ); and chest tenderness reproducing pain, 0.63 (95\% CI 0.45-0.80).

Figure 2 is a clinical depiction of the Vancouver Chest Pain Rule. In the derivation cohort, the rule would have identified all 165 patients with a 30-day ACS diagnosis while permitting 111 of 598 patients without ACS to be discharged safely within 2 hours with no follow-up, for a sensitivity of $100 \%$ and a specificity of $18.6 \%$. In the validation cohort, the rule would have identified 118 of 119 patients with ACS while allowing safe early discharge of 184 of 787 patients without ACS, for 99.2\% (95\% CI 95.4-100) sensitivity and $23.4 \%$ (95\% CI 20.4-26.5) specificity. Table 3 illustrates rule performance for the derivation and validation cohorts, respectively, and decision trees are shown in Appendices 3 and 4.

The rule missed no ACS patients in the derivation cohort but missed one patient with UA in the validation cohort. He was a 48-year-old male with no history of ACS or nitrate use, and his nonradiating pain was not reproducible with pressure. Although the initial ECG and troponin were normal, subsequent troponin assays were not done as the patient was taken to the catheterization lababoratory by Cardiology. The emergency physician referred him to Cardiology as he had documented prehospital hypotension. Angiography revealed a single 50\% mid-left anterior descending coronary artery lesion; he was discharged uneventfully 


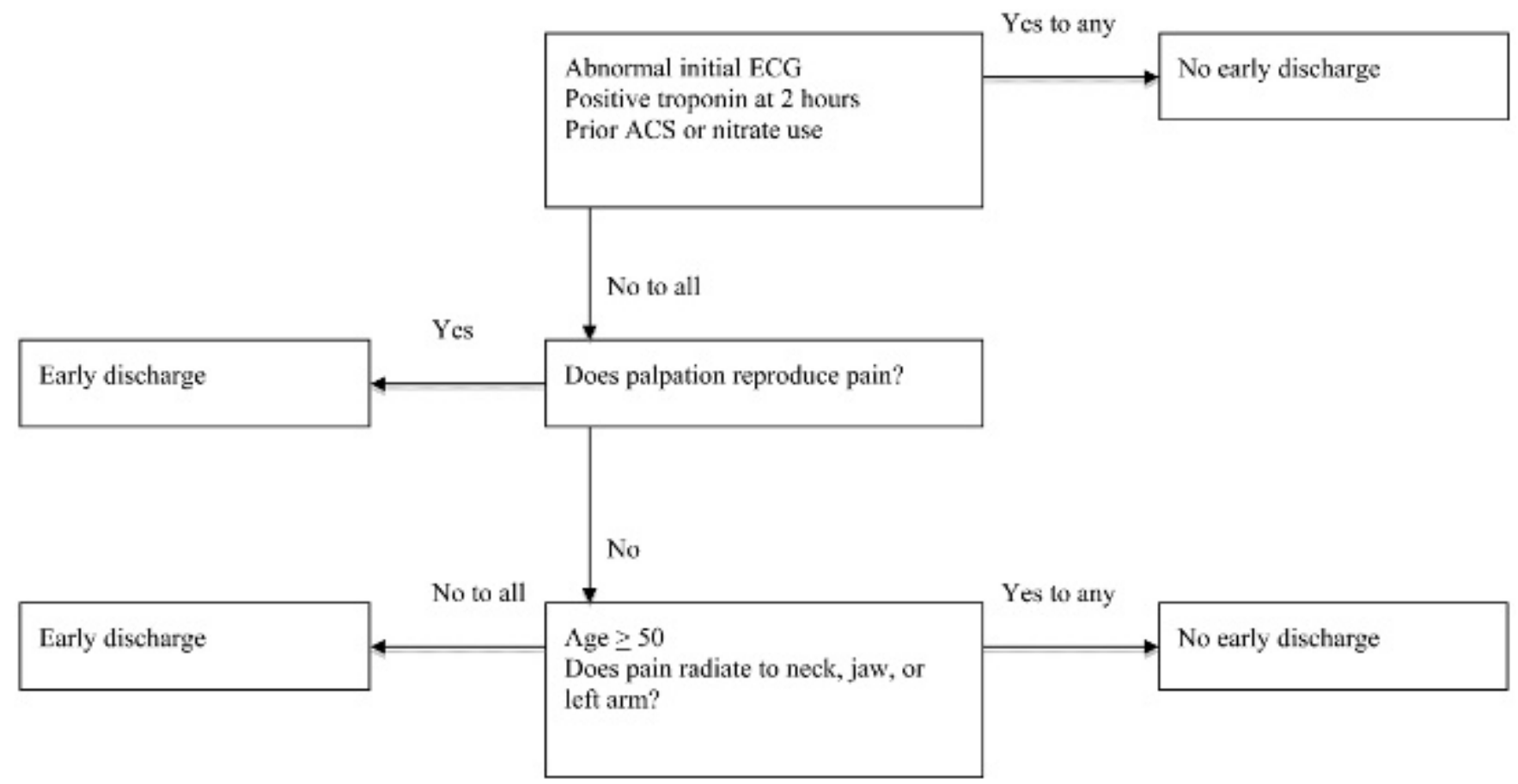

Figure 2. Vancouver Chest Pain Rule. ACS = acute coronary syndrome; ECG = electrocardiogram.

with a single stent. Although he may have met rule criteria for early discharge (no sequential cardiac testing was performed), he met study criteria for UA.

\section{DISCUSSION}

\section{Summary of major findings}

ED patients with acute ST elevation myocardial infarction and those with obvious noncardiac causes are often straightforward. In comparison, patients with nonspecific symptoms and low cardiac risk pose a significant diagnostic and cognitive burden on physicians while consuming substantial hospital resources and contributing to ED crowding. The Vancouver Chest Pain Rule assists clinicians by identifying a subset of chest pain patients (approximately one-fifth) who can be safely discharged within 2 hours with no further provocative cardiac testing while identifying more than 99\% of patients who have ACS. If applied across Canada, this could permit safe early discharge of up to 120,000 chest pain patients annually. Interestingly, some traditional cardiac risk factors, including hypertension, diabetes, and hyperlipidemia, were not included

\begin{tabular}{|c|c|c|c|}
\hline & Definite ACS & No definite ACS & Total \\
\hline \multicolumn{4}{|l|}{ Derivation cohort* } \\
\hline Unsuitable for early discharge & 165 & 487 & 652 \\
\hline Suitable for early discharge & 0 & 111 & 111 \\
\hline Total & 165 & 598 & 763 \\
\hline \multicolumn{4}{|l|}{ Validation cohort $^{\dagger}$} \\
\hline Unsuitable for early discharge & 118 & 603 & 721 \\
\hline Suitable for early discharge & 1 & 184 & 185 \\
\hline Total & 119 & 787 & 906 \\
\hline \multicolumn{4}{|c|}{$\begin{array}{l}\text { ACS }=\text { acute coronary syndrome. } \\
\text { *The sensitivity was } 100 \% \text {, and the specificity was } 18.6 \% \text {. The positive predictive value was } 25.3 \% \text {, and the negative predictive value was } \\
100 \% \text {. } \\
\text { 'The sensitivity was } 99.2 \%(95 \% \mathrm{Cl} 95.4-100) \text {, and the specificity was } 23.4 \%(95 \% \mathrm{Cl} 20.4-26.5) \text {. The positive predictive value was } 16.4 \% \\
(95 \% \mathrm{Cl} 13.8-19.2) \text {, and the negative predictive value was } 99.5 \%(95 \% \mathrm{Cl} 96.6-100.0) \text {. }\end{array}$} \\
\hline
\end{tabular}


in the final model; neither was the duration or quality (i.e., stabbing, burning) of pain.

\section{Comparison with other studies}

Many efforts have been devoted to rapidly identifying chest pain patients at low clinical risk of ACS who aresuitable for rapid discharge of patients. Guidelines developed by ACS-time-insensitive predictive instrument are structured toward disposition of low-risk patients, with only $21 \%$ of patients discharged home, but few at the 2-hour mark. ${ }^{10}$ The Erlanger protocol allows physicians to use objective criteria or clinical judgment to discharge or provocatively test patients at 2 hours from ED presentation but does not provide criteria to forego additional testing. ${ }^{11}$ Few studies have attempted to derive a clinical prediction rule to identify patients who can be safely discharged without further investigations, such as provocative testing or imaging. Bassan and colleagues developed a decision rule incorporating the clinical likelihood of ACS-patients selectively received investigations to confirm or refute the presence of disease, ensuring a varying "gold standard" for ACS diagnosis, ${ }^{12}$ whereas Fernandez Portales and colleagues had cardiologists enrol patients with a high clinical suspicion of $\mathrm{ACS}^{13}$; hence, the resulting rule may have limited generalizability in an ED setting.

Several rules for identifying patients who can be rapidly and safely discharged have been prospectively validated. Marsan and colleagues developed and validated a sensitive algorithm that involves minimal investigations in patients age 24 to 39 years. ${ }^{14}$ Lyons and colleagues validated the thrombosis in myocardial infarction (TIMI) score in ED patients with a high likelihood of ACS, ${ }^{15}$ but this may be difficult to extrapolate to a general ED population. Several studies have attempted to use Global Registry of Acute Coronary Events (GRACE) or TIMI scores to predict ACS, but 3 to $4 \%$ of the low-risk patients had a 30-day ACS outcome, ${ }^{15,16}$ a risk that may be unacceptable to clinicians. ${ }^{21}$ Many rules are hampered by selection bias, a suboptimal methodological design, or low sensitivity. ${ }^{28,29}$ There has been no widely used clinical prediction rule for safe early discharge of such patients, and the few rules that have been validated permit risk stratification of selected patients but do not allow for minimal investigations or early discharge.

Than and colleagues developed a protocol to classify ED chest pain patients based on a combination of the
TIMI score, ECG, and cardiac biomarkers. ${ }^{30}$ This promising strategy identified $9.8 \%$ of patients as low risk; this low-risk group had a 30 -day AE rate of $0.9 \%$. However, patients diagnosed with UA were included in the $\mathrm{AE}$ rate only if they were revascularized. Novel troponin assays appear to improve sensitivity for AMI diagnosi $^{31-33}$ and accurately stratify mortality ${ }^{34}$ in ED chest pain patients, but their utility in diagnosing $\mathrm{UA},{ }^{33}$ ruling out ACS, or permitting safe and rapid discharge in low-risk patients has not been demonstrated.

This rule uses reliable clinical criteria and widely available tests to define a group of patients who can be safely discharged at 2 hours without further observation or investigation. Designated outcomes were obtained by predefined explicit criteria, and an a priori blinded adjudication process resolved controversial diagnoses. The rule identifies ACS patients in a logically stepwise process, with most patients recognized by an abnormal ECG or cardiac biomarker, some diagnosed by historical elements, and a few identified by pain characteristics and age.

\section{LIMITATIONS}

This rule was derived and validated in a single tertiary care centre, and performance may vary in other settings. Our physicians were not asked to assess or interpret the rule during the prospective validation phase or describe their comfort with it; as a result, their willingness and ability to successfully use the rule, as well as the clinical sensibility of the rule, was not measured. The development cohort was enrolled around 10 years ago, but outcome definitions (see Table 1) reflect the current guidelines of care (2007 ACC/AHA guidelines). In addition, although we classified patients with a new LBBB as having AMI, newer data indicate that this may not be the case. ${ }^{35}$ (One patient in the validation group had a new LBBB but had a complete circumflex artery occlusion and received a stent during angiography.). Although the ACS rate decreased between the derivation and validation cohorts, identical outcomes were used to minimize verification bias. Some very sick patients, or those transferred rapidly for a cardiac procedure, may not have been enrolled, although such patients are typically not a diagnostic conundrum. We enrolled patients presenting with chest pain; therefore, our findings cannot be extrapolated to those with atypical symptoms such as weakness, nausea, or dyspnea. In 
particular, female patients, diabetics, and the elderly have been reported as having a higher percentage of alternate presenting complaints. ${ }^{36}$

Patients who were discharged from the ED were not required to have follow-up marker assays or mandatory provocative cardiac testing, and only approximately $40 \%$ of enrolled patients had these investigations. It is possible that we could have missed clinically silent ACS events or events for which patients did not seek medical care and thus did not receive additional testing or hospitalization. Our intensive patient follow-up was a reasonable proxy standard to capture clinically important outcomes. This rule cannot be endorsed for the detection of rare but potentially lethal causes of chest pain such as aortic dissection or pulmonary embolism.

A number of patients $(n=207)$ did not have sufficient data captured to participate in the validation cohort. Most often they were seen outside research assistant working hours and discharged with no cardiac biomarkers as emergency physicians were not mandated to obtain biomarkers or retain patients for at least 2 hours during the validation phase of the study. In comparison with those with data in the validation cohort, these patients were lower risk and younger (mean age 43 versus 60 years) with substantially lower rates of hypertension and hyperlipidemia than the overall cohort. None had previous ACS, and all had normal initial ECGs. All 207 patients were followed to 30 days: none died or suffered an ACS outcome or AE. Consequently, the exclusion of these patients did not falsely inflate rule sensitivity. Assuming that all would have had negative biomarkers, their inclusion would have increased specificity to $39.3 \%$ and sensitivity closer to $100 \%$.

\section{CONCLUSION}

The Vancouver Chest Pain Rule identifies a cohort of ED chest pain patients who can be safely discharged within 2 hours without further provocative cardiac testing. Although use of this rule would preserve health care resources with maintaining high sensitivity for ACS detection, further prospective external validation is required across other centres with comprehensive and uniform follow-up of all eligible and enrolled patients. In addition, the comfort of emergency physicians with the rule and their ability to interpret it successfully require assessment.
Acknowledgements: We are grateful for the support of Patti Barker, RN, Courtney Lawson, RN, Crystal-Ann Smith, RN, and Helen $\mathrm{Xu}, \mathrm{MD}$.

Competing interests: This study was funded by Canadian Institutes of Health Research grant MOP 53102 and the Heart and Stroke Foundation of British Columbia and Yukon.

\section{REFERENCES}

1. Pitts SR, Niska RW, Xu J, Burt CW. National Hospital Ambulatory Medical Care Survey: 2006 Emergency Department Summary. Natl Health Stat Report 2008 Aug $6 ;(7): 1-38$.

2. Goodacre S, Cross E, Arnold J, et al. The health care burden of acute chest pain. Heart 2005;91:229-30, doi:10.1136/ hrt.2003.027599.

3. Pope JH, Aufderheide TO, Ruthazer R, et al. Missed diagnoses of acute cardiac ischemia in the emergency department. $N$ Engl 7 Med 2000;342:1163-70, doi:10.1056/ NEJM200004203421603.

4. Christenson JM, Innes GD, McKnight RD, et al. Safety and efficiency of emergency department assessment of chest discomfort. CMAJ 2004;170:1803-7.

5. Karcz A, Korn R, Burke MC, et al. Malpractice claims against emergency physicians in Massachusetts: 1975-1993. Am 7 Emerg Med 1996;14:341-5, doi:10.1016/S07356757(96)90044-3.

6. Anderson JL, Adams CD, Antman EM, et al. American College of Cardiology/American Heart Association 2007 guidelines for the management of patients with unstable angina/nonST-elevation myocardial infarction. Available at: http:// circahajournals.org/cgi/reprint/circulationaha.107.185752 (accessed March 2, 2012).

7. Gibler WB, Cannon CP, Blomkalns AL, et al. Practical implementation of the guidelines for unstable angina/nonST-segment elevation myocardial infarction in the emergency department: a scientific statement from the American Heart Association Council on Clinical Cardiology (Subcommittee on Acute Cardiac Care), Council on Cardiovascular Nursing, and Quality of Care and Outcomes Research Interdisciplinary Working Group, in collaboration with the Society of Chest Pain Centers. Circulation 2005;111:2699-710, doi:10.1161/01.CIR.0000 165556.44271.BE.

8. Amsterdam E, Kirk J, Bluemke D, et al. Testing of low-risk patients presenting to the emergency department with chest pain: a scientific statement from the American Heart Association. Circulation 2010;122:756-76, doi:10.1161/CIR. 0b013e3181ec61df.

9. Hess EP, Brison RJ, Perry JJ, et al. Development of a clinical prediction rule for 30-day cardiac events in emergency department patients with chest pain and possible acute coronary syndrome. Ann Emerg Med 2011 Aug 31. [Epub ahead of print]

10. Selker HP, Beshansky JR, Griffith JL, et al. Use of the acute cardiac ischemia time-insensitive predictive instrument (ACI-TIPI) to assist with triage of patients with chest pain or other symptoms suggestive of acute cardiac ischemia. A multicenter, controlled clinical trial. Ann Intern Med 1998; 
129:845-55, doi:10.7326/0003-4819-129-11 Part 1-19981 2010-00002.

11. Fesmire FM, Hughes AD, Fody EP, et al. The Erlanger chest pain evaluation protocol: a one-year experience with serial 12-lead ECG monitoring, two-hour delta serum marker measurements, and selective nuclear stress testing to identify and exclude acute coronary syndromes. Ann Emerg Med 2002;40:584-94, doi:10.1067/mem.2002.129506.

12. Bassan R, Pimenta L, Scofano M, Soares JF. Accuracy of a neural diagnostic tree for the identification of acute coronary syndrome in patients with chest pain and no ST-segment elevation. Crit Pathw Cardiol 2004;3:72-8, doi:10.1097/ 01.hpc.0000128713.08115.54.

13. Fernandez Portales J, Perez Reyes F, Garcia Robles JA, et al. Risk stratification using combined ECG, clinical, and biochemical assessment in patients with chest pain without ST-segment evaluation. How long should we wait? Rev Esp Cardiol 2003;56:338-45.

14. Marsan RJ, Shaver KJ, Sease KL, et al. Evaluation of a clinical decision rule for young adult patients with chest pain. Acad Emerg Med 2005;12:26-31, doi:10.1111/j.1553-2712. 2005.tb01473.x.

15. Lyons R, Morris AC, Caesar D, et al. Chest pain presenting to the emergency department-to stratify risk with GRACE or TIMI? Resuscitation 2007;74:90-3, doi:10.1016/j.resuscitation. 2006.11.023.

16. Hess EP, Perry JJ, Calder LA, et al. Prospective validation of a modified thrombolysis in myocardial infarction risk score in emergency department patients with chest pain and possible acute coronary syndrome. Acad Emerg Med 2010;17: 368-75, doi:10.1111/j.1553-2712.2010.00696.x.

17. Greene J. The perils of low-risk chest pain: emergency physicians struggle to balance risk with overtesting. Ann Emerg Med 2010;56:25A-8A, doi:10.1016/j.annemergmed. 2010.08.021.

18. Pines JM, Pollack CV, Diercks DB, et al. The association between emergency department crowding and adverse cardiovascular outcomes in patients with chest pain. Acad Emerg Med 2009;16:17-25, doi:10.1111/j.1553-2712.2009. 00364.x.

19. Diercks DB, Roe MT, Chen AY, et al. Prolonged emergency department stays of non-ST-segment elevation myocardial infarction patients are associated with worse adherence to the American College of Cardiology/American Heart Association guidelines for management and increased adverse events. Ann Emerg Med 2007;50:489-96, doi:10. 1016/j.annemergmed.2007.03.033.

20. Christenson JM, Innes GD, McKnight RD, et al. A clinical prediction rule for early discharge of patients with chest pain. Ann Emerg Med 2006;47:1-10, doi:10.1016/j.annemergmed. 2005.08.007.

21. McGougan CK, Christenson JM, Innes GD, Raboud J. Emergency physicians' attitudes toward a clinical prediction rule for the identification and early discharge of low risk patients with chest discomfort. CFEM 2001;3:89-94.

22. Stiell IG, Wells GA. Methodologic standards for the development of clinical decision rules in emergency medicine. Ann Emerg Med 1999;33:437-47, doi:10.1016/S01960644(99)70309-4.
23. Laupacis A, Sekar N, Stiell IG. Clinical prediction rules: a review and suggested modifications of methodologic standards. JAMA 1997;277:488-94, doi:10.1001/jama.1997. 03540300056034.

24. Innes G, Murray M, Grafstein E. A consensus-based process to define standard national data elements for a Canadian emergency department information system. CFEM 2001;3: 277-84.

25. Beveridge R, Ducharme J, Janes L, et al. Reliability of the Canadian emergency department triage and acuity scale: inter-rater agreement. Ann Emerg Med 1999;34:155-9, doi:10.1016/S0196-0644(99)70223-4.

26. Hollander JE, Blomkalns AL, Brogan GX, et al. Standardized reporting guidelines for studies evaluating risk stratification of ED patients with potential acute coronary syndromes. Acad Emerg Med 2004;11:1331-40, doi:10.1197/ j.aem.2004.08.033.

27. Myocardial infarction redefined-a consensus document of the Joint European Society of Cardiology/American College of Cardiology Committee for the redefinition of myocardial infarction. Eur Heart $\mathcal{F}$ 2000;21:1502-13, doi:10.1053/ euhj.2000.2305.

28. Hess EP, Thiruganasambandamoorthy V, Wells GA, et al. Diagnostic accuracy of clinical prediction rules to exclude acute coronary syndrome in the emergency department setting: a clinical review. CFEM 2008;10:373-83.

29. Hess EP, Wells GA, Jaffe A, Stiell IG. A study to derive a clinical decision rule for triage of emergency department patients with chest pain: design and methodology. $B M C$ Emerg Med 2008;8:3, doi:10.1186/1471-227X-8-3.

30. Than M, Cullen L, Reid CM, et al. A 2-h diagnostic protocol to assess patients with chest pain symptoms in the Asia-Pacific region (ASPECT): a prospective observational validation study. Lancet 2011;377:1077-84, doi:10.1016/ S0140-6736(11)60310-3.

31. Keller T, Zeller T, Peetz D, et al. Sensitive troponin I assay in early diagnosis of acute myocardial infarction. $N$ Engl 7 Med 2009;361:868-77, doi:10.1056/NEJMoa0903515.

32. Reichlin T, Hochholzer W, Bassetti S, et al. Early diagnosis of myocardial infarction with sensitive troponin assays. $N$ Engl 7 Med 2009;361:858-67, doi:10.1056/NEJMoa0900428.

33. Keller T, Zeller T, Ojeda F, et al. Serial changes in highly sensitive troponin I assay and early diagnosis of myocardial infarction. FAMA 2011;306:2684-93, doi:10.1001/jama.2011. 1896.

34. Mills NL, Churchhouse A, Lee KK, et al. Implementation of a sensitive troponin I assay and risk of recurrent myocardial infarction and death in patients with suspected acute coronary syndrome. $7 A M A$ 2011;305:1210-6, doi:10.1001/ jama.2011.338.

35. Chang AM, Shofer FS, Tabas JA, et al. Lack of association between left bundle-branch block and acute myocardial infarction in symptomatic ED patients. Am 7 Emerg Med 2009;27:916-21, doi:10.1016/j.ajem.2008.07.007.

36. Canto JG, Shlipak MG, Rodgers W, et al. Prevalence, clinical characteristics, and mortality among patients with myocardial infarction presenting without chest pain. $7 \mathrm{AMA}$ 2000;283:3223-9, doi:10.1001/jama.283.24.3223. 


\section{Appendix 1. Candidate predictor variables collected for original derivation cohort}

Pain and associated symptom characteristics

Age*

Gender*

Was chest pain present on arrival?*

Did chest pain continue at rest?*

Number of episodes in previous 24 hours

Number of episodes in previous week

Maximum duration of previous episode*

Diaphoresis

Weakness

Dyspnea

Nausea

Vomiting

Palpitations

Syncope

Dizziness/lightheadedness

Radiation to left arm/shoulder*

Radiation to right arm/shoulder*

Radiation to back

Radiation to neck*

Radiation to jaw*

Radiation to abdomen

Radiation to lower limb

Pain in centre of chest*

Pain in left lateral chest

Pain in right anterior chest

Pain in right lateral chest

Most prominent character of pain*

Heavy chest discomfort*

Maximum discomfort level*

Pain diagnosed in past as $\mathrm{AMI}^{*}$

Pain diagnosed in past as angina

Pain diagnosed in past as either AMI or angina

Pain worse with deep breaths*

Pain worse in different positions

Did pain improve within 5 minutes of nitroglycerin?*

Did pain improve within 5 minutes of oxygen?

Did pain improve within 5 minutes of liquid antacid?

Within first 2 hours, did pain relieve and not return?

Within first 2 hours, did pain continue?*

Within first 2 hours, did pain come and go?*

Physical examination

Chest clear

Crackles at bases

Crackles to scapulae

Wheezes

Heart sounds normal

Systolic murmur

Diastolic murmur

\section{Appendix 1. Continued}

$\mathrm{S}_{3}$ present

$\mathrm{S}_{4}$ present

Carotid pulses equal with no bruit

Radial pulses equal

Physician palpation in area of maximum chest discomfort reproducing pain*

Right arm systolic pressure

Left arm systolic pressure

Systolic pressure $<90 \mathrm{~mm} \mathrm{Hg}$ in 2 hours*

Bradycardia $<50$ beats per minute in 2 hours*

Tachycardia $>100$ beats per minutes in 2 hours*

Risk factors

Regular smoker*

History of diabetes*

History of hypertension*

History of hyperlipidemia*

Parent or sibling with heart attack $<65$ years

Recreational substance use

Alcohol use in past 24 hours

Cocaine use in past 48 hours

ECG findings

First ECG result normal*

First ECG with only T-wave flattening*

Aggregate of ECGs with no ischemic changes*

2-hour ST segment monitoring (any ST elevation or ST depression)*

Laboratory markers

0-hour troponin*

2-hour troponin*

Difference in troponin at 0 and 2 hours*

Cardiovascular history

Previous angina*

Previous AMI*

Previous cardiac arrest*

Previous angiogram with lesion $>50 \%$ *

Previous angiogram with lesion $<50 \%$ *

Previous $\mathrm{PCl}^{*}$

Previous peripheral vascular surgery

Previous congestive heart failure*

Previous CABG*

Previous stroke*

Previous TIA*

Previous ventricular arrhythmias

Previous atrial arrhythmias

Previous unknown arrhythmias

Medications

Previous ASA*

Previous other antiplatelet agents*

Previous ACEI*

Previous digitalis*

Previous beta-blocker* 


\begin{tabular}{l}
\hline Appendix 1. Continued \\
Previous warfarin* \\
Previous low-molecular-weight heparin* \\
Previous nitrates* \\
Previous lipid-lowering agents* \\
Previous antiarrhythmics* \\
Previous diuretics* \\
Previous other antihypertensives* \\
ACEI = angiotensin-coverting enzyme inhibitor; $A C S=$ acute coronary syndrome; $A E=$ \\
adverse event; AMI = acute myocardial infarction; $\mathrm{ASA}=$ acetylsalicylic acid; $\mathrm{CABG}=$ \\
coronary artery bypass grafting; ECG = electrocardiogram; $\mathrm{PCI}=$ percutaneous \\
coronary intervention; TIA = transient ischemic attack. \\
*Variable included in validation cohort.
\end{tabular}

\section{Appendix 2. Univariate predictive statistics for predictors used in the rule}

Predictor ACS (\%) No ACS (\%) Odds ratio* $(95 \% \mathrm{Cl})$

Number of patients

Abnormal initial $\mathrm{ECG}^{\dagger}$

Age $\geq 50 \mathrm{yr}$

Previous AMI

Previous angina

Previous nitrate use

Previous AMI, angina, or nitrate use

Pain radiates to jaw

Pain radiates to neck

Pain radiates to left arm

No pain increase with inspiration

Palpitation does not reproduce pain

Pain radiates and does not increase with inspiration or palpitation

Troponin increase within first 2 hours Ischemic ECG changes within first 2 hours

ST-T ECG changes within first 2 hours if initial ECG normal

$\begin{array}{ccc}165 & 598 & \\ 57.6 & 22.6 & 4.7(3.2-6.7) \\ 96.4 & 72.2 & 12.8(5.1-31.7) \\ 35.8 & 21.0 & 2.1(1.4-3.1) \\ 53.9 & 31.0 & 2.6(1.8-3.8) \\ 48.5 & 23.8 & 3.0(2.1-4.4) \\ 66.7 & 40.6 & 2.9(2.0-4.3) \\ 24.9 & 12.3 & 2.4(1.5-3.7) \\ 35.2 & 24.3 & 1.7(1.1-2.5) \\ 57.0 & 43.4 & 1.7(1.2-2.5) \\ 78.2 & 64.2 & 2.0(1.3-3.1) \\ 94.3 & 80.5 & 4.0(2.0-9.3) \\ 48.4 & 25.6 & 2.7(1.9-4.0) \\ 35.5 & 15.1 & 3.1(2.0-4.7) \\ 68.9 & 33.5 & 1.2(0.4-2.9) \\ 42.9 & 20.7 & 2.9(1.7-4.8) \\ & \end{array}$




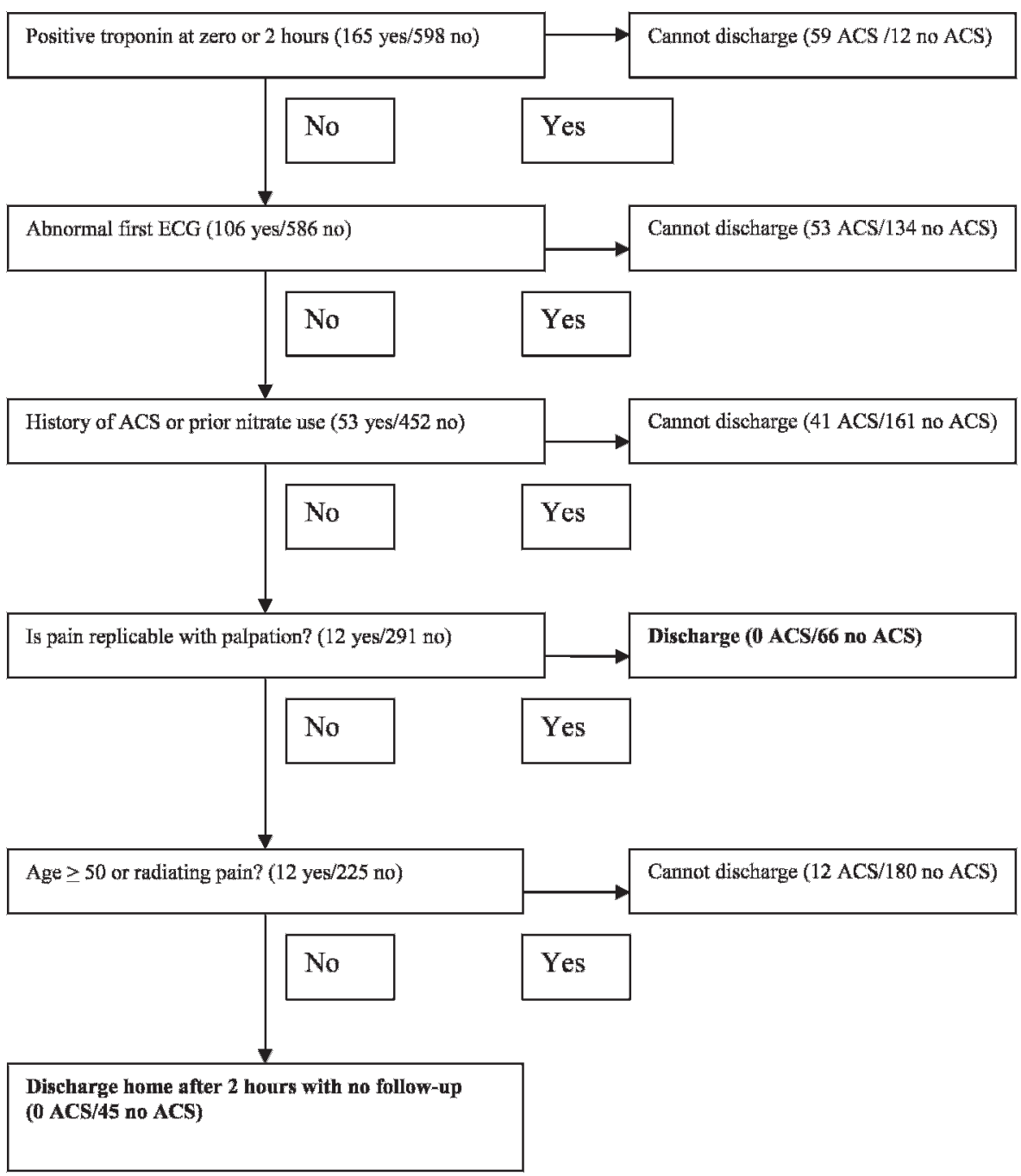

Appendix 3. Decision tree for derivation cohort $(n=763)$. ACS = acute coronary syndrome; ECG = electrocardiogram. 


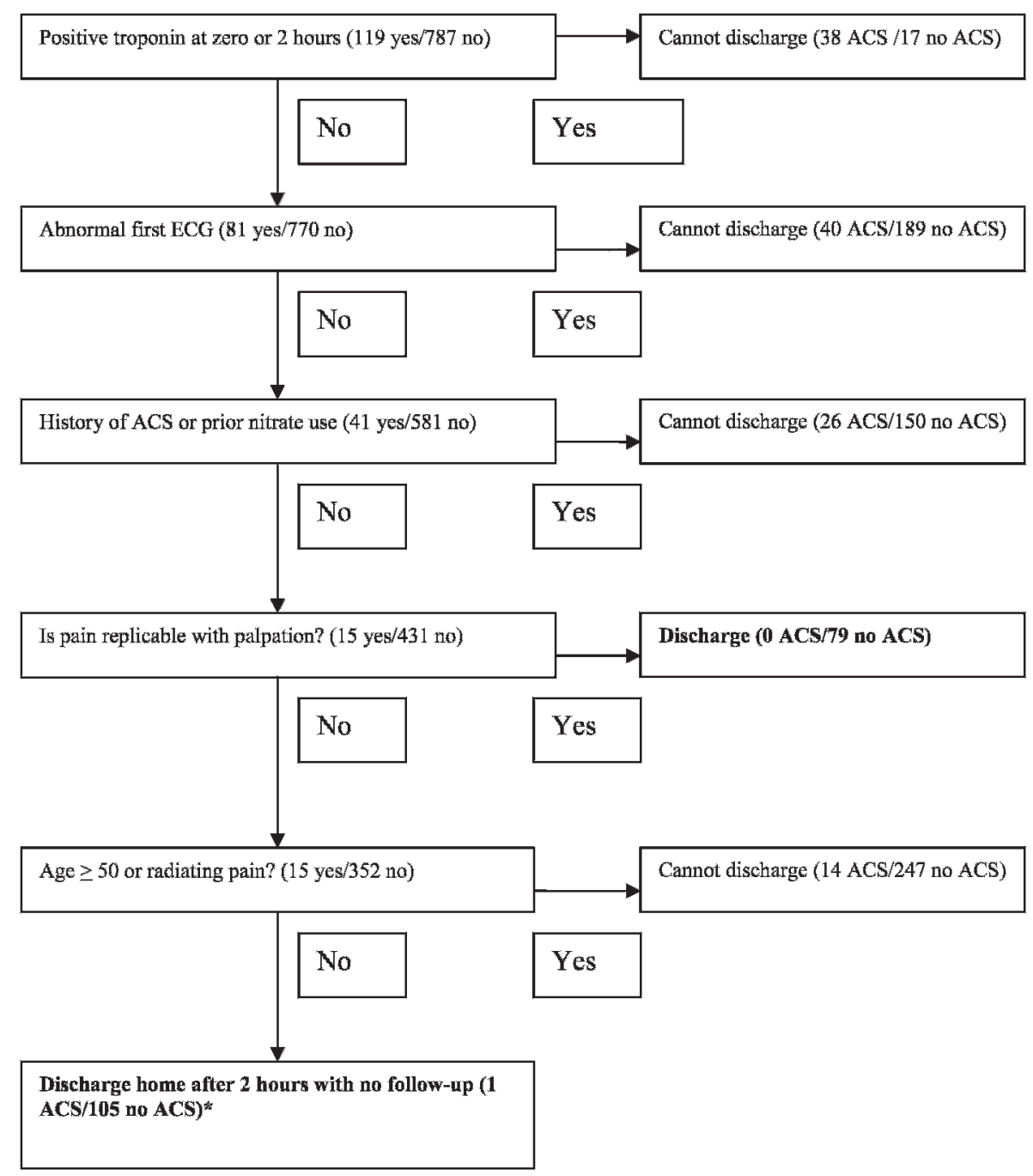

Appendix 4. Decision tree for validation cohort $(n=906$; 831 patients with complete data, 75 patients evaluable despite incomplete data). ACS = acute coronary syndrome; ECG = electrocardiogram. 